\section{Sentences for use in memory research}

\section{JULIE R. BRANNAN and CAMERON J. CAMP \\ University of New Orleans, New Orleans, Louisiana}

To investigate the effects of effort toward comprehension on subsequent recall, Auble, Franks, and Soraci (1979) generated a series of 40 sentences which varied in comprehensibility. We presented these sentences along with 60 new sentences to 122 undergraduates at the University of New Orleans. Sentences were rated for comprehensibility both before and after cue presentation. Sentences which appear most useful for research are discussed.

Auble, Franks, and Soraci (1979) created 40 sentences for use in studies on the effects of effort toward comprehension on memory. Twenty of these sentences were generally comprehended without a cue provided by the experimenter (easy sentences), and 20 were generally comprehended only after a cue had been provided (difficult sentences). Auble and Franks (1983) later used these stimuli to study spreading activation models of semantic memory.

While using these sentences (Brannan \& Camp, 1984; Monroe, Camp, Markley, \& Kramer, 1982), we noted that not all easy sentences were comprehended before (or even after) cue presentation, whereas some difficult sentences were understood before cue presentation (and some were never comprehended). Auble and Franks encountered similar problems (1983, pp. 398-399). As a result, their data and ours were analyzed on the basis of itemby-item judgments.

It seemed useful to determine whether the original classification of sentences as easy or difficult would replicate in a different population, setting, and time. It also seemed appropriate to use a larger sample of respondents and to try to discover additional sentences for use in research.

\section{Method}

Subjects. One hundred twenty-two students at the University of New Orleans served as participants.

Stimulus items. Two sets of 50 sentences each were constructed. Each set contained 20 items from the Auble et al. (1979) stimuli list and 30 original items of the same form. Ten easy and 10 difficult sentences from the $\mathrm{Au}$ ble et al. stimuli were presented in a random order as Items 11 through 30 in each set. Each set was available in two forms, one containing the sentences without cues, and a second form with appropriate cues.

The authors would like to thank Bruce Dorval and an anonymous reviewer for their helpful suggestions regarding earlier drafts of this manuscript. J. R. Brannan's mailing address is: Psychology Department, University of New Orleans, Lakefront, New Orleans, LA 70148.
Rating task. Participants were asked to read the first set of 50 sentences, marking on a response sheet whether or not they understood each sentence. When finished, they were given the alternate form containing the appropriate cues, and they marked on a different response sheet whether they now understood the sentences. The procedure was then repeated on the second set of sentences. The order of presentation was counterbalanced.

Criteria. Like Auble et al. (1979), we used the following criteria for easy or difficult sentences shown without cues: (1) for an easy sentence, at least $67 \%$ of the subjects understood the sentence in isolation, and (2) for a difficult sentence, no more than $33 \%$ of subjects understood the sentence in isolation. However, given the large number of participants in our sample $(N=122)$ compared to that of Auble et al. $(N=19)$, we assumed that a criterion which excluded any sentence not understood after cue presentation by any single participant would be too stringent; therefore, an $80 \%$ cutoff point was selected as being indicative of sentence comprehensibility after cue presentation for both types of sentences.

\section{Results and Discussion}

Using our criteria, 30 of the original 40 Auble et al. (1979) sentences qualified as either easy or difficult. Of the 60 newly created sentences, 25 met the criteria for easy sentences and 6 met the criteria for difficult sentences. We offer these as substitutes for Auble et al.'s 10 original sentences which did not meet the criteria. Samples of the ratings for Auble et al.'s 40 stimuli and the 31 newly generated sentences are found in Table $1 .{ }^{1}$

The sentences that met our criteria are likely to elicit shifts to comprehension at the intended times (before or after cues). However, these outcomes also support the contention of Auble and Franks (1983) that comprehension of sentences must be empirically verified, since only 12 of the 61 sentences meeting our criteria were understood by $100 \%$ of the subject sample after cue presentation. Researchers should verify the time at which comprehension occurs (before or after cue presentation) on an item-by-item basis. Once this is done, investigators should be able to use these materials effectively in a wide variety of memory tasks involving comprehension, context effects, ambiguity, sudden insight, and inferential reasoning.

\section{REFERENCES}

Auble, P. M., Franks, J. J., \& SoraCi, S. A. (1979). Effort toward comprehension: Elaboration or "'aha!'? Memory \& Cognition, 7, 426-434.

Auble, P., \& Franks, J. J. (1983). Sentence comprehension processes. Journal of Verbal Learning \& Verbal Behavior, 22, 395-405.

Brannan, J. R., \& Camp, C. J. (1984, May). Picture this: Visual cue 
Table 1

Examples of Stimuli

\begin{tabular}{|c|c|c|c|}
\hline \multirow[b]{2}{*}{ Sentence (Cue) } & \multicolumn{2}{|c|}{ Before Cues } & \multirow{2}{*}{$\frac{\text { After Cues }}{\text { Brannan \& Camp }}$} \\
\hline & Auble et al. & Brannan \& Camp & \\
\hline
\end{tabular}

EXAMPLES OF STIMULI MEETING OUR CRITERIA

Easy Sentences

(Auble et al.)

The teacher stopped writing because the stick crumbled. (chalk)

$$
95 \%
$$

$82 \%$

$99 \%$

(Brannan \& Camp)

The man was late because his wrist was bare. (wristwatch)

$83 \%$

Difficult Sentences

(Auble et al.)

The woman could not see because the blade was bent. (windshield wipers)

(Brannan \& Camp)

The man stayed cold because the book was wet. (matches)

EXAMPLES OF STIMULI NOT MEETING OUR CRITERIA

Sentence Not Attaining $80 \%$ or More Comprehension After the Cue

The breakfast was excellent

because the thread was sticky.

$0 \%$

$7 \%$

(spider web)

"Difficult" Sentence Not Attaining 33\% or Less Comprehension After the Cue

The audience cheered because the five balls rotated.

$21 \%$

$46 \%$

$99 \%$

(juggling)

"Easy" Sentence Not Attaining 67\% or More Comprehension Before the Cue

The man got well because the

slicing was successful.

$100 \%$

$55 \%$

$97 \%$ (operation)

Note-Examples and data are taken from "Effort toward comprehension: Elaboration or "aha!"? by P. M. Auble, J. J. Franks, and S. A. Soraci, 1979, Memory \& Cognition, 7, 426-434, and from the present study (Brannan \& Camp).

superiority effects in the recall of ambiguous sentences. Paper presented at the annual meeting of the Louisiana Psychological Association, Lafayette, LA.

Monroe, R. M., Camp, C. J., Markley, R. P., Kramer, J. J. (1982, October). "Aha" and aging: Effects of an everyday insight experience on recall. Paper presented at the annual meeting of the Midwestern Psychological Association, Minneapolis, MN.

\section{NOTE}

1. A complete listing of the ratings for Auble et al.'s (1979) 40 stimuli and the 31 newly generated sentences can be obtained from J. R. Brannan.

(Revision accepted for publication April 8, 1986.) 